\title{
New Obesity Index Associated with Fitness Among Korean Adults
}

\author{
Nam Lyong Kang \\ Department of Nanomechatronics Engineering, Pusan National University, Miryang, Republic of Korea \\ Email address: \\ nlkang@pusan.ac.kr

\section{To cite this article:} \\ Nam Lyong Kang. New Obesity Index Associated with Fitness Among Korean Adults. International Journal of Clinical and Experimental \\ Medical Sciences. Vol. 7, No. 1, 2021, pp. 5-12. doi: 10.11648/j.ijcems.20210701.12
}

Received: December 21, 2020; Accepted: December 29, 2020; Published: January 15, 2021

\begin{abstract}
Background: The aim of this paper was to introduce a new obesity index associated with fitness and determine effective exercises for reducing the obesity in common Korean adults using a new kind of waist-to-height ratio (WHT2R). Objectives: This cross-sectional study was performed using the public-use releases of the 2017 survey of national physical fitness by the Korea Institute of Sport Science of the Korea Sports Promotion Foundation. Method: The correlation coefficients were calculated by correlation analyses between the obesity indices and four fitness tests for comparison. The lump mean values (LMVs) were introduced to find the association between the WHT2R and fitness tests using a linear regression method and to use as an indicator for the effective control of obesity. Result: The WHT2R is more associated with the fitness than the other indices and the correlation with fitness tests was interpreted more simply using the LMV of the WHT2R rather than that of the body mass index. Setting the goal of exercise using the WHT2R was easier and more convenient than using the body mass index. Conclusion: The WHT2R can be used as a possible contender for predicting fitness and is an effective indicator for the reduction of abdominal obesity.
\end{abstract}

Keywords: Waist-to-Height Ratio, Abdominal Obesity, Fitness, Correlation Coefficient, Lump Mean Value, Body Mass Index

\section{Introduction}

Fitness is important because physical activity or exercise can reduce the level of obesity associated with cardiovascular diseases, such as hypertension [1-4]. Lavie et al. examined the adverse effects of physical inactivity on the prevention of cardiovascular diseases in a study on the pathophysiological effects of weight gain on the cardiovascular system [5]. Therefore, an effective anthropometric index for evaluating obesity is necessary for an enhancement of fitness and as an effective means of preventing and treating obesity. Hydrostatic densitometry is considered a good tool for assessing obesity, but it is inconvenient. More convenient and less expensive anthropometric indices, such as the body mass index (BMI) and waist-to-height ratios, have been used as alternative indicators of obesity and cardiovascular diseases.

The BMI is internationally accepted as a standard for assessing obesity [6-9]. The advantages of the BMI are that it is not intrusive and easy to calculate with acceptable accuracy. On the other hand, abdominal obesity may be tied more closely to metabolic risks than the BMI [10-14]. Therefore, many studies have considered the waist-to-height ratio (WHtR), which is defined as the waist circumference (WC) divided by the height, as an indicator of abdominal obesity or a parameter to predict the risk of cardiovascular diseases [15-18]. Nevill et al. introduced a new waist-to height ratio (WHT.5R) as the WC divided by the square root of the height and found it to be the best anthropometric index of the cardiometabolic risk [19]. Bustamante et al. suggested that the reciprocal Ponderal index is a more appropriate index to assess fatness [20]. Krakauer et al. developed a body shape index $(\mathrm{ABSI})$, which is defined as $\mathrm{WC} /\left(\mathrm{BMI}^{2 / 3} \times\right.$ height $\left.^{1 / 2}\right)$, based on the WC adjusted for height and weight, and used it as a substantial risk factor for premature mortality [21].

Many researchers have considered the BMI, ABSI, WHT.5R, and WHTR as indices for measuring abdominal obesity and predicting the risk of cardiovascular diseases [15, 19, 22]. Among these, the WHT.5R and WHtR are used widely as useful indices for predicting the risk of cardiovascular diseases because the BMI cannot represent the central fat distribution, and the 
waist circumference cannot differentiate between the visceral fat and subcutaneous fat [23-25].

This paper presents a new waist-to height ratio, WHT2R, which is defined as the WC divided by the square of the height, for assessing abdominal obesity associated with fitness. The WC is used instead of weight in the BMI to indicate abdominal obesity. The body mass index, body shape index, and two other waist-to-height ratios (WHT.5R, WHTR) are considered as possible contenders for the WHT2R. The correlation coefficients between the different indices and fitness tests were calculated to determine which among the BMI, ABSI, and three waist-toheight ratios (WHT.5R, WHtR, and WHT2R) has the best association with fitness. The association between WHT2R and fitness was also investigated per generation. On the other hand, it was impossible to find the dependence of fitness on the index individually because the distribution of fitness with respect to the index is quite complicated. Therefore, this study used the lump mean value (LMV), which was introduced by the present author, to determine the BMI for best fitness [26]. The WHT2Rdependence of the fitness test scores was investigated using the LMVs and a linear regression method, and the results were then compared with the BMI-dependence of the fitness test scores.

\section{Methods and Materials}

\subsection{Study Design and Participants}

This cross-sectional study was performed using the publicuse releases of the 2017 survey of national physical fitness (ISBN 979-11-952035-6-7) by the Korea Institute of Sport Science of the Korea Sports Promotion Foundation (KSPO). Statistics Korea (National Statistics No 113004) approved the collection of data and samples from the cohorts participating in the study. All research participants provided written consent to take part. The final study cohort comprised of 2082 males and 2038 females from a total of 4296 subjects aged 19 to 64 years. The subjects were excluded if they had extreme values of the five indices or four fitness tests (Table 1).

Table 1. Ranges of BMI, ABSI, WHT.5R, WHtR, WHT2R, Sit-up, SLJ, 20-m MSSR, and 10-m SR considered.

\begin{tabular}{|c|c|c|c|c|}
\hline \multirow{2}{*}{ Quantity [unit] } & \multicolumn{2}{|l|}{ Men } & \multicolumn{2}{|c|}{ Women } \\
\hline & Min. & Max. & Min. & Max. \\
\hline BMI $\left[\mathrm{kg} / \mathrm{m}^{2}\right]$ & 18.0 & 35.7 & 16.0 & 34.9 \\
\hline ABSI $\left[\mathrm{cm}^{11 / 6} / \mathrm{kg}^{2 / 3}\right]$ & 0.58 & 0.96 & 0.56 & 1.02 \\
\hline WHT.5R $\left[\mathrm{cm}^{0.5}\right]$ & 5.03 & 9.17 & 4.57 & 8.62 \\
\hline WHTR $\times 10^{-2}[$ none $]$ & 38.5 & 69.3 & 36.1 & 67.6 \\
\hline $\mathrm{WHT} 2 \mathrm{R} \times 10^{-4}\left[\mathrm{~cm}^{-1}\right]$ & 22.0 & 39.9 & 21.1 & 43.6 \\
\hline Sit-up [times/minute] & 2 & 72 & 2 & 65 \\
\hline $\mathrm{SLJ}[\mathrm{cm}]$ & 61 & 280 & 50 & 223 \\
\hline 20-m MSSR [times] & 3 & 100 & 2 & 82 \\
\hline $10-\mathrm{m} \mathrm{SR}[\mathrm{sec}]$ & 8.5 & 19.3 & 9.5 & 21.4 \\
\hline
\end{tabular}

$\dagger$ Abbreviations: $\mathrm{BMI}=$ body mass index; $\mathrm{ABSI}=\mathrm{a}$ body shape index; WHT.5R=waist circumference divided by the square root of the height; WHtR=waist-to-height ratio; WHT2R=waist circumference divided by the square of the height; SLJ=standing long jump; 20-m MSSR=20-m multistage shuttle run; $10-\mathrm{m} \mathrm{SR}=10-\mathrm{m}$ shuttle run.

The fitness tests considered were sit-up, standing long jump (SLJ), 20-m multi-stage shuttle run (20-m MSSR), and 10-m shuttle run (10-m SR) tests. The sit-up, SLJ, and 20-m MSSR tests measure the level of muscular endurance, speed, and cardiorespiratory endurance, respectively. The 10-m SR test measures the speed and agility (See Ref. 26 for details).

\subsection{Analysis}

This study examined which among the BMI, ABSI, WHT.2R, WHTR, and WHT2R has the best association with the fitness tests and was carried based on many studies, where the WC was more associated with the cardiometabolic mortality than the BMI, and height had an inverse association with the mortality [27-29]. The WHT2R is defined using the WC instead of weight in BMI to consider the abdominal obesity as follows:

$$
\text { WHT2R } \equiv \frac{\text { waist circumference }}{{\text { (height })^{2}}^{2}}
$$

The correlation coefficients between the indices and fitness tests were calculated using correlation analyses in Microsoft Excel 2014, and linear regression was carried out using Sigmaplot 14. The distribution of fitness with respect to WHT2R is quite complicated. Therefore, the lump mean values were considered to investigate the dependence of fitness on the WHT2R. Male and female subjects were divided into groups containing approximately 105 and 95 subjects with successive WHT2Rs except near the two endpoints.

\section{Results}

\subsection{Correlation Between the Indices and Fitness Tests}

Figure 1 shows the correlation coefficients $(r)$ between the indices and fitness tests. A more significant correlation coefficient indicates a stronger correlation between the two values. Therefore, Figure 1 shows that the WHT2R has a stronger correlation with all the fitness tests than the other four indices. Sit-ups were negatively correlated with all five indices for both males and females. This means that the number of situps performed in the test decreases with increasing index. The BMI increases with increasing weight, while ABSI, WHT.5R, WHtR, and WHT2R increase with increasing waist circumstance, and the muscular endurance decreases with decreasing number of sit-ups. Therefore, muscular endurance decreases with increasing weight or waist circumstance.

The SLJ performance was negatively correlated with all five indices for both males and females. This means that the SLJ score decreases with increasing index. Therefore, the speed decreases with increasing weight or waist circumstance because it decreases with decreasing SLJ score. The 20-m MSSR performance was also negatively correlated with all five indices for both males and females. This means that the 20-m MSSR performance decreases with increasing index. Therefore, cardiorespiratory endurance decreases with increasing weight or waist circumstance because the cardiorespiratory endurance decreases with decreasing 20-m MSSR performance. In contrast, the $10-\mathrm{m}$ SR time was 
positively correlated with all five indices for both males and females. This means that the 10 -m SR time increases with increasing index. Hence, the speed and agility decrease with

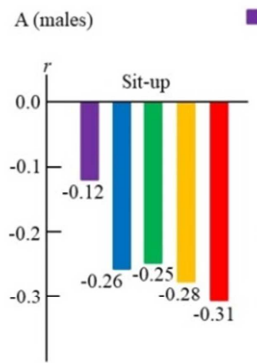

aMI $\quad$ ABSI $\quad$ WHtR

increasing weight or waist circumstance because an increase in the 10-m SR time means a decrease in speed and agility.
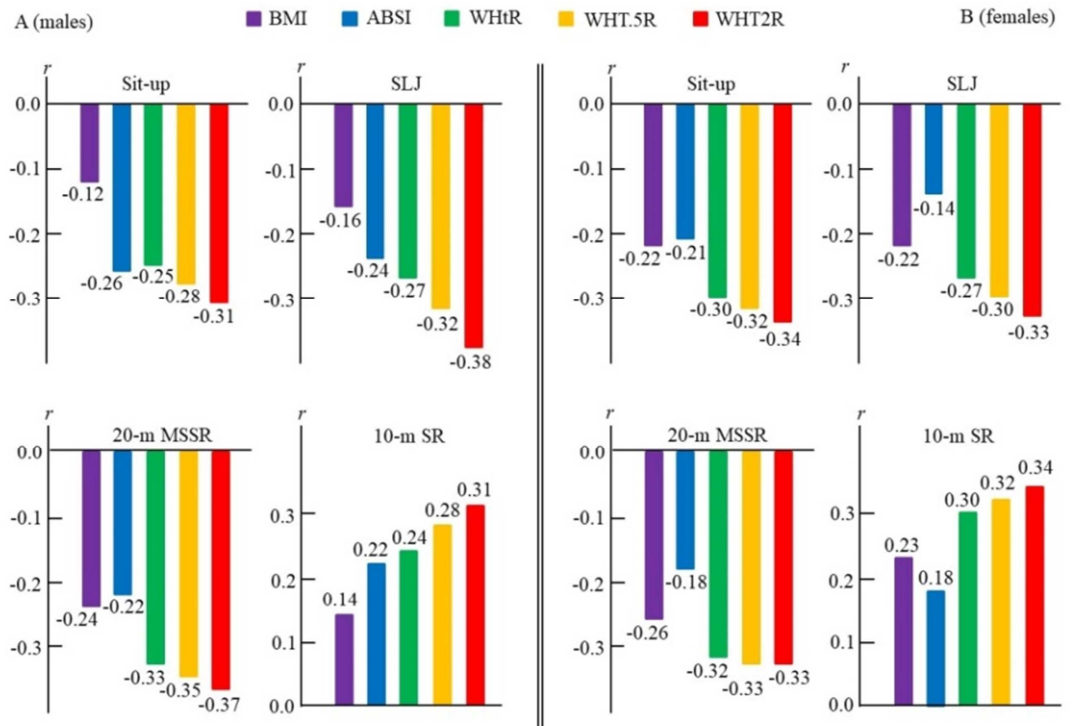

Figure 1. A. Correlation coefficients $(r)$ between the indices and fitness tests for males. B. Correlation coefficients $(r)$ between the indices and fitness tests for females.

\subsection{Correlation Between the WHT2R and Fitness Tests Per Generation}

Figure 2 shows the correlation coefficients $(r)$ between the WHT2Rs and fitness tests per generation.

The correlations for males were highest in those in their $30 \mathrm{~s}$ in all the fitness tests and then decreased with age. The correlations for females increased with age and were highest in those in their 50s in all fitness tests. In Korea, every healthy male youth must perform military service, where they exercise regularly. Therefore, the increase in abdominal obesity and the decrease in fitness by the change in life pattern after military service, as well as aging, can explain the highest correlations for males in their $30 \mathrm{~s}$. The decreases in the correlations in males older than 40 years can be interpreted by the increase in the concern for health and aging. This means that the correlations increase as male age due to the increase in abdominal obesity and the decrease in fitness, but these increases are curtailed by the decrease in abdominal obesity and increase in fitness due to physical activity or exercise (Figure 2A).
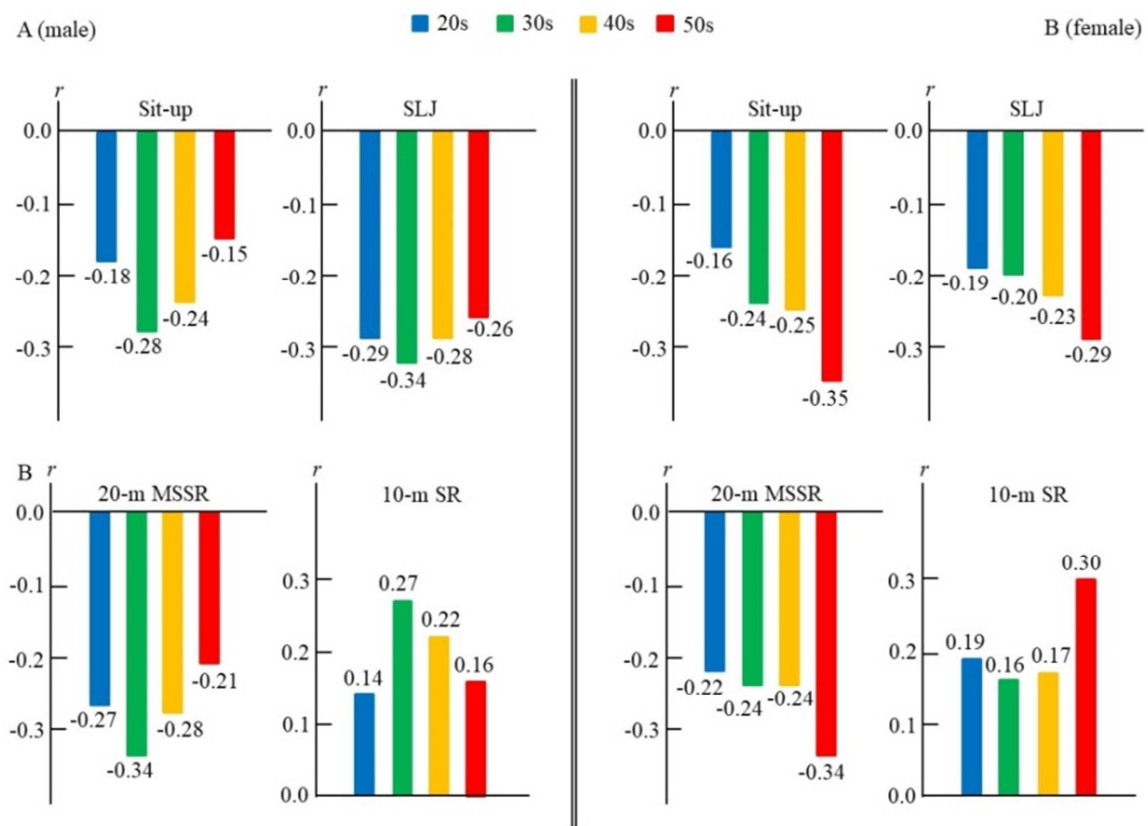

Figure 2. A. Correlation coefficients between the WHT2R and fitness tests for males per generation. B. Correlation coefficients between the WHT2R and fitness tests for females per generation. 
The reasons why the correlations increase with age for females can be attributed to the increase in age for childbirth and concerns for body shape as well as aging. The correlations for females are relatively small in their 20 s - 40s owing to the distorted association between fitness and body shape occurred because they reduced their abdominal obesity by controlling food intake for a good body shape but they did not increase their fitness by exercise. On the other hand, the correlation increases from 40 years due to the increase in abdominal obesity and the decrease in fitness after childbirth (Figure 2B).

\subsection{Analysis Using the Lump Mean Values}

The dependence of fitness on WHT2R was investigated using the lump mean values (LMVs) (Tables 3 and 4 in Appendix). Each lump was composed of subjects with successive WHT2R except near the two endpoints and the number of subjects contained in each lump was slightly different according to WHT2R because the number of subjects with the same WHT2R is not uniform and decreases toward the two endpoints because of a lack of subjects. Figure 3 shows that the LMV of the sit-up test decreases linearly with increasing LMVs of WHT2R among males. On the other hand, the LMVs of the sit-up test increased as the LMVs of BMI among males increased to $\mathrm{BMI}_{\max }$ and then decreased as the BMI increased from $\mathrm{BMI}_{\max }$ showing a Gaussian distribution, where $\mathrm{BMI}_{\max }\left(=23.4 \times 10^{-4} \mathrm{~cm}^{-1}\right)$ denotes the BMI for the best fitness ${ }^{26}$ and it was smaller than the mean value of BMI $\left(\mathrm{BMI}_{\text {mean }}=24.76 \times 10^{-4} \mathrm{~cm}^{-1}\right)$. The condition for the best fitness could be obtained using the LMV of the BMI because the LMVs of the sit-up test with respect to the LMVs of the BMI was fitted to a Gaussian curve, as shown in the inset, while the LMVs of the sit-up test with respect to the LMVs of WHT2R can be fitted to a straight line, as shown in Figure 3, which is expressed as:

$$
y(x)=m x+c
$$

where $x, c, m$, and $y(x)$ are the LMV of WHT2R, a constant, slope, and LMVs of the four fitness tests. Variation of the LMVs of fitness tests with respect to that of the LMVs of WHT2R increases with increasing $m . m$ is positive for a positive correlation and negative for a negative correlation. The figure shows the two parameters defined in Eq. (2), $m$ and $c$. The values at the two end points are unstable because of a lack of subjects. The discrepancies between the black circles and solid blue line can be reduced if a sufficiently large number of subjects are available. Figure 3 shows that the goodness-of-fit for linear regression is sufficient because the coefficients of determination $\left(r^{2}\right)$ are sufficiently large.

The LMVs of the 10-m SR test with respect to the LMVs of WHT2R among males can be fitted to a straight line, as shown in Figure 4, whereas the LMV of the 10-m SR test decreased as the LMVs of the BMI among males increases to $\mathrm{BMI}_{\text {max }}$ and then increased with further increases in BMI, as in an inverse Gaussian distribution as shown in the inset. The LMVs of SLJ and 20-m MSSR tests with respect to WHT2R can be fitted to the straight lines in the same manner. Table 1 lists the results, including those for women. The results show that the LMVs of all fitness tests can be fitted better to a straight line than a Gaussian curve using the LMV of the WHT2R rather than using the LMV of the BMI. This means that setting the goal of exercise using the WHT2R is easier and more convenient than using the BMI. The smallest WHT2R is the WHT2R for the best fitness. On the other hand, WHT2R is not applicable to establish an exercise aim of people with very weak fitness because it does not include the muscle, whereas the BMI is applicable because it includes the muscle and fat in the weight [26] if other effects, such as the lung capacity, affecting the fitness score are neglected.

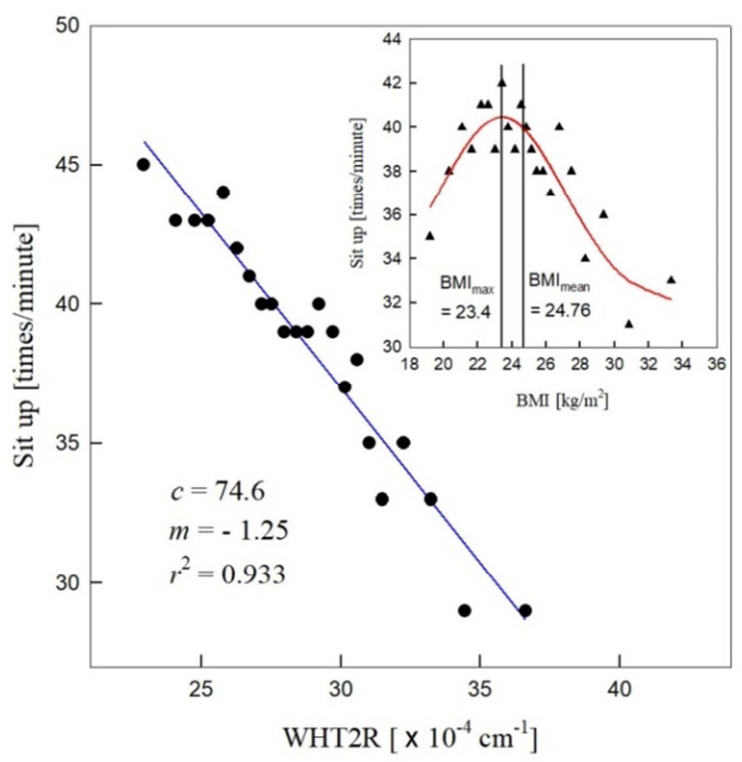

Figure 3. Black circles are the LMVS of the sit-up test for the LMVS of the $W H T 2 R$ for males, and the solid blue line denotes the straight line for the black circles fitted to Eq. (2). The inset shows a previous result for the BMI [26].

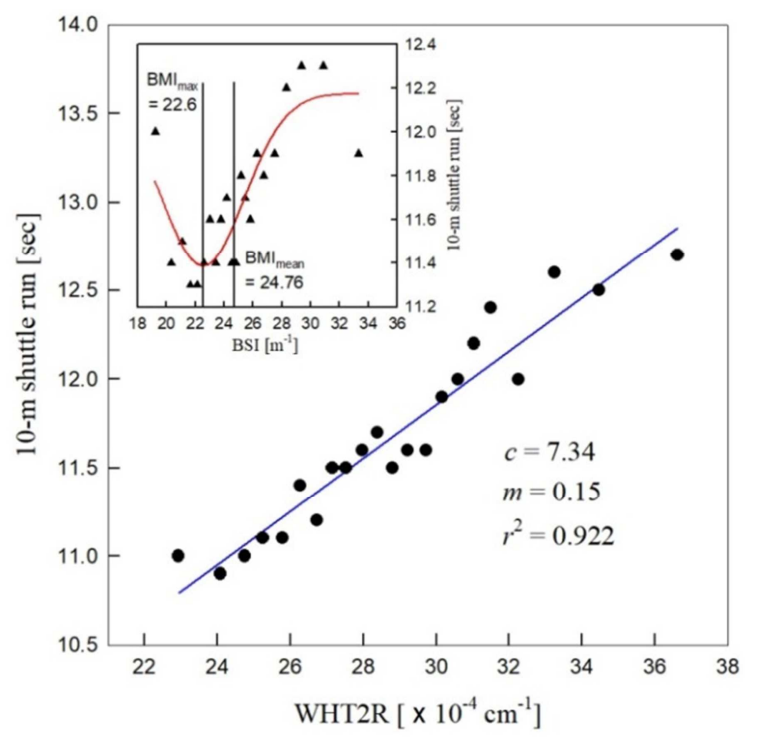

Figure 4. Black circles are the LMVs of the 10-m SR test for the LMVs of the $W H T 2 R$ for males and the solid blue line denotes the straight line for the black circles fitted to Eq. (2). The inset shows the result for BMI [26]. 
Table 2. $m$ and $c$ for fitting the lump mean values of the fitness tests $(y)$ with respect to the lump mean values of the WHT2R $(x)$ to a straight line, $y(x)=$ $m x+c$.

\begin{tabular}{lllllllll}
\hline & Male & \multicolumn{9}{c}{ Female } \\
\cline { 2 - 8 } & Sit-up & SLJ & 20-m MSSR & 10-m SR & Sit-up & SLJ & 20-m MSSR \\
\hline$m$ & -1.25 & -3.5 & -2.09 & 0.15 & -0.99 & -2.19 & -0.92 & 0.15 \\
$c$ & 74.6 & 298.4 & 97.4 & 7.34 & 55.1 & 208.5 & 48.4 & 0.9 \\
$r^{2}$ & 0.933 & 0.922 & 0.933 & 0.922 & 0.883 & 0.937 & 0.949 & 0.41 \\
\hline
\end{tabular}

Note: The dimension of $c$ is same as that of each fitness test, and the dimension of $m$ is the same as the dimension of each fitness test divided by cm. $r^{2}=$ coefficients of determination. [Units: sit-up (times/minute), SLJ (cm), 20-m MSSR (times), 10-m SR (second)].

\section{Discussion}

Overall, the sit-up, SLJ, and 20-m MSSR performance decreased while the $10-\mathrm{m}$ SR increased as the BMI, ABSI, WHT.5R, WHtR, and WHT2R were increased. Therefore, the sit-up, SLJ, and 20-m MSSR performance were negatively correlated with the indices, whereas the $10-\mathrm{m} \mathrm{SR}$ was positively correlated with them. The correlation coefficients between the BMI and the four fitness tests for females were larger than those for males. The four fitness tests were the most weakly correlated with the BMI for males and the ABSI for females. On the other hand, the WHT.5R, WHtR, and WHT2R were more associated with all the fitness tests than the BMI and ABSI, and there was a stronger correlation between all the fitness tests and the WHT2R than the WHT.5R and WHtR for both males and females. Therefore, the WHT2R is a more accurate index for explanation of relation between the fitness and abdominal obesity than the BMI, ABSI, WHT.5R, and WHtR.

The waist-to-height ratio $\left(\mathrm{WC} /\right.$ height $\left.^{b}\right)$ becomes more associated with all the fitness tests as the scaling exponent $(b)$ increases (Figure 1) but the WHT3R, which is defined as the WC divided by the cube of the height $(b=3)$, did not show a stronger association with all of the fitness tests than the WHT2R. The correlation coefficients $(r)$ between the WHT3R and fitness tests were $r=-0.31$ (sit-up), -0.40 (SLJ), -0.35 (20-m MSSR), and 0.31 (10-m SR) for males, and $r=-0.33$ (sit-up), -0.34 (SLJ), -0.32 (20-m MSSR), and $0.34(10-\mathrm{m} \mathrm{SR})$ for females. Therefore, the waist-toheight ratio $\left(\mathrm{WC} /\right.$ height $\left.^{b}\right)$ with $b$ values between 2 and 3 will have the best association with all of the fitness tests. This needs to be studied more using the allometric model to identify the optimal body size and shape [20].

Although the WHT2R has the best association with all of the fitness tests, it does not mean that it has an acceptable goodness of fit because the coefficient of determination $\left(r^{2}\right)$ is quite low $\left(r^{2} \sim 0.1\right)$. On the other hand, the associations for other four indices (BMI, ABSI, WHT.5R, WHtR) were smaller than that of the WHT2R. Therefore, the WHT2R can contribute more effectively to explaining relation between the fitness and abdominal obesity than the other indices if additional factors, such as sex, smoking, diabetes, blood pressure, and other factors are considered and if more advanced statistical methods are used. Although the reasons for the strong correlations for males in their 30 s and females in their 50s were attributed to military service for males and childbirth for females, more study considering aging, cardiopulmonary function, morphological characteristic, and muscular strength will be needed.

The correlation of the LMV of the fitness with that of the WHT2R was fitted to a simple straight line in the present study, whereas that with the LMV of the BMI was fitted to a Gaussian curve in the previous study [26]. Therefore, the correlation with fitness was interpreted more simply using the LMV of the WHT2R rather than that of the BMI. The desired WHT2R value could be obtained simply and uniquely from the minimum values of a straight line corresponding to the best values of the sit-up, SLJ, 20-m MSSR, and 10-m SR test scores, while they were different according to the best values of the each fitness test given by the Gaussian curves [26].

\section{Conclusion}

This study showed that waist circumference rather than body weight should be used in a body index to assess abdominal obesity. Furthermore, this study showed that the waist circumference is more appropriate as an anthropometric index when it is divided by the square of the height than when it is divided by the height or square root of the height. A new waist-to-height ratio (WHT2R) introduced in this study was a more effective anthropometric index for predicting fitness than the body mass index and other waistto-height ratios. Setting the goal of exercise using the WHT2R was easier and more convenient than using the BMI because the fitness with respect to the WHT2R could be fitted to a simple straight line while the fitness with respect to the BMI was fitted to the Gaussian curve. On the other hand, the BMI has merit in that it would be applicable to establish the exercise goal of people with poor fitness. Therefore, it is expected that the WHT2R could be used as a more effective ratio to determine the abdominal obesity and establish an exercise aim if the BMI is used in a complementary manner. On the other hand, Solon et al. found that a single index (FFI: fit-fat index) combining fitness (CRF: cardiorespiratory fitness) and fatness (WHtR) provides a more comprehensive indication of incident diabetes when compared to fitness or fatness alone [30]. It is expected that the WHT2R introduced in this paper could be used for the single index instead of WHtR. The WHT2R and LMV may be used as a useful indicator for fitness and abdominal obesity of other races with similar physiques to set their exercise aims. A study for predicting high blood pressure and wide pulse pressure using the present method will be done in the near future. 


\section{Appendix}

Table 3. Lump mean values of the WHT2R ( $\times 10^{-4} \mathrm{~cm}^{-1}$ ), Sit-up (times/minute), SLJ (cm), 20-m MSSR (times), and 10-m SR (second) for males.

\begin{tabular}{|c|c|c|c|c|c|c|c|c|c|c|c|}
\hline \multirow{2}{*}{$\mathbf{N}$} & \multicolumn{3}{|l|}{ WHT2R } & \multicolumn{2}{|l|}{ Sit-up } & \multicolumn{2}{|l|}{ SLJ } & \multicolumn{2}{|c|}{ 20-m MSSR } & \multicolumn{2}{|c|}{ 10-m SR } \\
\hline & Range & Mean & SD & Mean & SD & Mean & SD & Mean & SD & Mean & SD \\
\hline 60 & $21.98-23.49$ & 22.93 & 0.43 & 45 & 11.5 & 218 & 26.8 & 47 & 19.3 & 11.0 & 1.36 \\
\hline 78 & $23.53-24.49$ & 24.08 & 0.28 & 43 & 13.1 & 218 & 28.0 & 48 & 20.2 & 10.9 & 1.34 \\
\hline 81 & $24.50-24.98$ & 24.75 & 0.14 & 43 & 12.3 & 212 & 23.3 & 47 & 20.1 & 11.0 & 1.17 \\
\hline 91 & $25.00-25.49$ & 25.24 & 0.15 & 43 & 11.6 & 212 & 28.5 & 46 & 17.2 & 11.1 & 1.63 \\
\hline 100 & $25.50-26.03$ & 25.79 & 0.16 & 44 & 12.1 & 210 & 25.9 & 48 & 19.4 & 11.1 & 1.28 \\
\hline 103 & $26.04-26.49$ & 26.27 & 0.14 & 42 & 12.1 & 203 & 28.5 & 41 & 16.9 & 11.4 & 1.59 \\
\hline 107 & $26.50-26.94$ & 26.72 & 0.13 & 41 & 11.3 & 204 & 28.4 & 40 & 19.0 & 11.2 & 1.32 \\
\hline 107 & $26.95-27.34$ & 27.15 & 0.12 & 40 & 13.9 & 203 & 28.4 & 43 & 18.8 & 11.5 & 1.58 \\
\hline 106 & $27.35-27.70$ & 27.52 & 0.11 & 40 & 10.4 & 204 & 23.5 & 40 & 16.6 & 11.5 & 1.47 \\
\hline 106 & $27.71-28.19$ & 27.97 & 0.14 & 39 & 11.6 & 198 & 24.7 & 37 & 15.3 & 11.6 & 1.29 \\
\hline 107 & $28.20-28.60$ & 28.39 & 0.12 & 39 & 11.4 & 196 & 23.1 & 38 & 17.9 & 11.7 & 1.37 \\
\hline 106 & $28.61-29.00$ & 28.80 & 0.11 & 39 & 12.3 & 199 & 26.6 & 36 & 16.6 & 11.5 & 1.25 \\
\hline 107 & $29.01-29.46$ & 29.22 & 0.12 & 40 & 10.4 & 202 & 22.5 & 38 & 16.3 & 11.6 & 1.50 \\
\hline 107 & $29.47-29.94$ & 29.72 & 0.14 & 39 & 12.6 & 196 & 27.9 & 37 & 17.8 & 11.6 & 1.45 \\
\hline 107 & $29.95-30.39$ & 30.15 & 0.13 & 37 & 11.7 & 192 & 25.0 & 34 & 14.9 & 11.9 & 1.41 \\
\hline 106 & $30.40-30.81$ & 30.59 & 0.13 & 38 & 10.0 & 192 & 23.7 & 32 & 13.8 & 12.0 & 1.33 \\
\hline 101 & $30.82-31.27$ & 31.03 & 0.14 & 35 & 11.6 & 187 & 26.8 & 31 & 14.8 & 12.2 & 1.50 \\
\hline 98 & $31.28-31.78$ & 31.49 & 0.14 & 33 & 11.8 & 178 & 26.9 & 30 & 12.1 & 12.4 & 1.65 \\
\hline 95 & $31.80-32.68$ & 32.25 & 0.26 & 35 & 10.5 & 183 & 27.8 & 31 & 13.6 & 12.0 & 1.44 \\
\hline 87 & $32.69-33.79$ & 33.24 & 0.32 & 33 & 11.6 & 184 & 29.1 & 25 & 13.4 & 12.6 & 1.79 \\
\hline 68 & $33.81-35.18$ & 34.46 & 0.39 & 29 & 11.1 & 177 & 22.5 & 26 & 13.3 & 12.5 & 1.30 \\
\hline 54 & $33.52-39.87$ & 36.62 & 1.27 & 29 & 12.3 & 177 & 26.5 & 24 & 11.5 & 12.7 & 1.55 \\
\hline
\end{tabular}

† Abbreviations: $\mathrm{N}=$ the number of subjects in a lump; Mean=the lump mean values for the ranges; $\mathrm{SD}=$ standard deviation.

Table 4. Lump mean values of the WHT2R ( $\times 10^{-4} \mathrm{~cm}^{-1}$ ), Sit-up (times/minute), SLJ (cm), 20-m MSSR (times), and 10-m SR (second) for females.

\begin{tabular}{|c|c|c|c|c|c|c|c|c|c|c|c|}
\hline \multirow{2}{*}{$\mathbf{N}$} & \multirow{2}{*}{$\begin{array}{l}\text { WHT2R } \\
\text { Range }\end{array}$} & \multirow[b]{2}{*}{ Mean } & \multirow[b]{2}{*}{ SD } & \multicolumn{2}{|l|}{ Sit-up } & \multicolumn{2}{|l|}{ SLJ } & \multicolumn{2}{|c|}{ 20-m MSSR } & \multicolumn{2}{|c|}{ 10-m SR } \\
\hline & & & & Mean & SD & Mean & SD & Mean & SD & Mean & SD \\
\hline 55 & $21.06-24.18$ & 23.29 & 0.78 & 30 & 12.4 & 153 & 29.3 & 25 & 12.9 & 13 & 1.45 \\
\hline 64 & $24.22-25.09$ & 24.66 & 0.27 & 29 & 11.1 & 154 & 21.7 & 26 & 12.7 & 13.1 & 1.49 \\
\hline 85 & $25.11-25.89$ & 25.49 & 0.23 & 31 & 12.3 & 156 & 26.7 & 27 & 11 & 13 & 1.34 \\
\hline 90 & $25.90-26.55$ & 26.23 & 0.18 & 30 & 10.8 & 149 & 21.7 & 24 & 10.8 & 13.3 & 1.35 \\
\hline 94 & $26.56-27.14$ & 26.84 & 0.17 & 29 & 10.6 & 150 & 22.3 & 24 & 11.1 & 13.5 & 1.43 \\
\hline 94 & $27.16-27.64$ & 27.39 & 0.15 & 31 & 9.76 & 150 & 20.3 & 24 & 11.3 & 13.5 & 1.43 \\
\hline 96 & $27.65-28.15$ & 27.88 & 0.15 & 26 & 12 & 147 & 23.8 & 22 & 10.3 & 13.7 & 1.77 \\
\hline 95 & $28.16-28.64$ & 28.4 & 0.16 & 26 & 12.1 & 146 & 26.5 & 23 & 10.7 & 13.5 & 1.62 \\
\hline 96 & $28.65-29.07$ & 28.87 & 0.13 & 27 & 10.7 & 146 & 28.4 & 21 & 9.74 & 13.9 & 1.74 \\
\hline 95 & $29.09-29.47$ & 29.29 & 0.11 & 27 & 11.5 & 145 & 23.9 & 22 & 10.8 & 13.6 & 1.39 \\
\hline 94 & $29.48-30.02$ & 29.75 & 0.16 & 26 & 12.4 & 144 & 27 & 22 & 12.2 & 13.7 & 1.49 \\
\hline 95 & $30.03-30.45$ & 30.24 & 0.14 & 24 & 10.9 & 143 & 22.3 & 21 & 10.1 & 13.9 & 1.59 \\
\hline 96 & $30.46-30.94$ & 30.72 & 0.14 & 24 & 10.9 & 139 & 24 & 20 & 11.5 & 14.1 & 1.85 \\
\hline 94 & $30.96-31.48$ & 31.21 & 0.15 & 26 & 10.6 & 142 & 20.4 & 20 & 9.35 & 13.8 & 1.3 \\
\hline 96 & $31.49-31.96$ & 31.73 & 0.14 & 27 & 10.7 & 141 & 23.2 & 19 & 9.04 & 14.1 & 1.65 \\
\hline 94 & $31.97-32.51$ & 32.25 & 0.16 & 23 & 10.7 & 138 & 22.5 & 19 & 9.46 & 14.3 & 1.61 \\
\hline 94 & $32.54-33.05$ & 32.79 & 0.15 & 21 & 10.7 & 134 & 24.5 & 17 & 8.04 & 14.4 & 1.51 \\
\hline 94 & $33.07-33.67$ & 33.37 & 0.18 & 23 & 12.2 & 140 & 23.5 & 18 & 11.3 & 14 & 1.58 \\
\hline 90 & $33.68-34.53$ & 34.08 & 0.25 & 20 & 10.8 & 131 & 25.9 & 16 & 10.4 & 14.7 & 1.73 \\
\hline 92 & $34.54-35.54$ & 34.96 & 0.27 & 21 & 9.95 & 134 & 23.8 & 16 & 6.95 & 14.5 & 1.81 \\
\hline 88 & $35.56-36.79$ & 36.17 & 0.37 & 17 & 9.17 & 125 & 24.1 & 15 & 6.79 & 14.8 & 1.64 \\
\hline 80 & $36.81-38.38$ & 37.6 & 0.49 & 19 & 10.7 & 129 & 22.4 & 13 & 5.62 & 14.8 & 1.65 \\
\hline 67 & $38.42-43.58$ & 40.03 & 1.24 & 15 & 8.21 & 118 & 25 & 13 & 7.1 & 15.6 & 1.97 \\
\hline
\end{tabular}

† Abbreviations: $\mathrm{N}=$ the number of subjects in a lump; Mean=the lump mean values for the ranges; $\mathrm{SD}=$ standard deviation. 


\section{Abbreviations}

WHT2R: Waist-to height ratio; LMV: Lump mean value; BMI: Body mass index; WC: Waist circumference.

\section{Authors' Contributions}

NLK is responsible for the idea, design, implementation, and analysis of the study. The author read and approved the final manuscript.

\section{Availability of Data and Materials}

All data generated or analysed during this study are included in this published article.

\section{Ethics Approval and Consent to Participate}

Ethics approval was not required as this study was performed using the public-use releases of a nationally representative cross-sectional survey. This article does not contain any studies with human or animals participants performed by the author.

\section{Competing Interests}

The authors declare that they have no competing interests.

\section{Acknowledgements}

The author would like to thank the Korea Sports Promotion Foundation for providing the data from the 2017 survey of national physical fitness. This research did not receive any specific grants from funding agencies in the public, commercial, or non-profit sectors.

\section{References}

[1] Lakka TA, Venalainen JM, Rauramaa R, Salonen R, Tuomilehto J, Salonen JT. Relation of leisure-time physical activity and cardiorespiratory fitness to the risk of acute myocardial infraction in Men. New Engl J Med. 1994; 330 (22): $1549-54$.

[2] Dubbert PM, Carithers T, Sumner AE, Barbour KA, Clark BL, Hall JE, Crook ED. Obesity, physical activity, and risk for cardiovascular disease. Am J Med Sci. 2002; 324 (3): 116-26.

[3] Carr MC, Brunzell JD. Abdominal obesity and dyslipidemia in the metabolic syndrome: importance of type 2 diabetes and familial combined hyperlipidemia in coronary artery disease risk. J Clin Endocr Metab. 2004; 89 (6): 2601-7.

[4] Matsuzawa Y. Establishment of a concept of visceral fat syndrome and discovery of adiponectin. P Jap Acad B-Phys. 2010; 86 (2): 131-41

[5] Lavie CJ, Ozemek C, Carbone S, Katzmarzyk PT, Blair SN.
Sedentary behavior, exercise, and cardiovascular health. Circ Res. 2019; 124 (5): 799-815.

[6] Hu G, Barengo NC, Tuomilehto J, Lakka TA, Nissinen A, Jousilahti P. Relationship of Physical Activity and Body Mass Index to the Risk of Hypertension: A Prospective Study in Finland. Hypertension. 2004; 43 (1): 25-30.

[7] Raustorp A, Pangrazi RP, Stähle A. Physical activity level and body mass index among schoolchildren in south-eastern Sweden. Acta Paediatr. 2004; 93 (3): 400-04.

[8] Pasco JA, Holloway KL, Dobbins AG, Kotowicz MA, Williams LJ, Brennan SL. Body mass index and measures of body fat for defining obesity and underweight: a cross-sectional, populationbased study. BMC Obesity. 2014; 23: 1-9.

[9] Kawai VK, Nwosu SK, Kurnik D, Harrell FE, Stein CM. Variants in BMI-associated genes and adrenergic genes are not associated with gestational weight trajectory. Obesity. 2019; 27 (7): 1184-9.

[10] Blair D, Habicht JP, Sims EA, Sylwester D, Abraham S. Evidence for an increased risk for hypertension with centrally located body fat and the effect of race and sex on this risk. Am J Epidemiol. 1984; 119 (4): 526-39.

[11] Kaplan NM. The deadly quartet. Upper-body obesity, glucose intolerance, hypertriglyceridemia, and hypertension. Arch Intern Med. 1989; 149 (7): 1514-20.

[12] Smalley KJ, Knerr AN, Kendrick ZV, Colliver JA, Owen OE. Reassessment of body mass indices. Am J Clin Nutr. 190; 52 (3): 405-8.

[13] Hsieh SD, Yoshinaga H. Waist/height ratio as a simple and useful predictor of coronary heart disease risk factors in women. Internal Med. 1995; 34 (12): 1147-52.

[14] Lee JS, Aoki K, Kawakubo K, Gunji A. A study on indices of body fat distribution for screening for obesity. J Occup Health. 1995; 37 (1): 9-18.

[15] Hsieh SD, Yoshinaga H, Muto T. Waist-to-height ratio, a simple and practical index for assessing central fat distribution and metabolic risk in Japanese men and women. Int J Obesity. 2003; 27 (5): 610-6.

[16] Shen S, Lu Y, Qi H, Li F, Shen Z, Wu L, Yang C, Wang L, Shui K, Yao W, Qiang D, Yun J, Zhou L. Waist-to-height ratio is an effective indicator for comprehensive cardiovascular health. Sci Rep-UK. 2017; 7: 43046.

[17] Choi JR, Koh SB, Choi E. Waist-to-height ratio index for predicting incidences of hypertension: the ARIRANG study. BMC Public Health. 2018; 18 (1): 767.

[18] Vasquez F, Correa-Burrows P, Blanco E, Gahagan S, Burrows R. A waist-to-height ratio of 0.54 is a good predictor of metabolic syndrome in 16-year-old male and female adolescents. Pedia Res. 2019; 85 (3): 269-74.

[19] Nevill AM, Duncan MJ, Lahart IM, Scandercock GR. Scaling waist girth for differences in body size reveals a new improved index associated with cardiometabolic risk. Scan J Med Sci Spor. 2017; 27 (11): 1470-6.

[20] Valdivia AB, Maia J, Nevill A. Identifying the ideal body size and shape characteristics associated with children's physical performance tests in Peru. Scan J Med Sci Spor. 2015; 25 (2): e155-65. 
[21] Krakauer NY, Krakauer JC. A new body shape index predicts mortality hazard independently of body mass index. PLoS ONE. 2012; 7 (7): e39504.

[22] Zhu S, Wang Z, Heshka S, Heo M, Faith MS, Heymsfield SB. Waist circumference and obesity-associated risk factors among whites in the third National Health and Nutrition Examination Survey: clinical action thresholds. Am J Clin Nutr 2002; 76 (4): 743-9.

[23] Taylor RW, Keil D, Gold EJ, Goulding A. Body mass index, waist circumference girth, and waist-to hip circumference ratio as indexes of total and regional adiposity in women: evaluation using receiver operating characteristic curve. Am J Clin Nutr. 1998; 67 (1): 44-9.

[24] Lu M, Ye W, Adami HO, Weiderpass E. Prospective study of body size and risk for stroke amongst women below age 60 . J Intern Med. 2006; 260 (5): 442-50.

[25] Ashwell M, Gunn P, Gibson S. Waist-to-height ratio is a better screening tool than waist circumference and BMI for adult cardiometabolic risk factors: systematic review and meta-analysis. Obes Rev. 2012; 13 (3): 275-86.
[26] Hwang S, Cho YS, Kang NL. Association between body mass index and fitness in Korean adults according to the lump mean value and Gaussian fitting. Med Hypotheses. 2020; 135: 109471.

[27] Langenberg C, Shipley MJ, Batty GD, Marmot MG. Adult socioeconomic position and the association between height and coronary heart disease mortality: findings from 33 years of follow-up in the Whitehall Study. Am J Public Health. 2005; 95 (4): 628-32.

[28] Seidell JC. Waist circumference and waist/hip ratio in relation to all-cause mortality, cancer and sleep apnea. Eur J Clin Nutr 2010; 64 (1): 35-41.

[29] Petursson H, Sigurdsson JA, Bengtsson C, Nilson TIL, Getz L. Body configuration as a predictor of mortality: comparison of five anthropometric measures in a 12 year follow-up of the Norwegian HUNT 2 study. PLoS ONE 2011; 6 (10): e26621.

[30] Sloan RA, Haaland BA, Sawada SS, Lee IM, Sui X, Lee DC, Ridouane Y, Müller-Riemenschneider F, Blair SN. A Fit-Fat Index for Predicting Incident Diabetes in Apparently Healthy Men: A Prospective Cohort Study. PLoS ONE 2016; 11 (6): e0157703. 